\title{
Immunogenicity and therapeutic effects of a Mycobacterium tuberculosis rv2190c DNA vaccine in mice
}

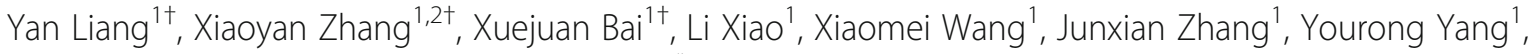 \\ Jinying Song ${ }^{1}$, Lan Wang ${ }^{1}$ and Xueqiong $\mathrm{Wu}^{1 *}$
}

\begin{abstract}
Background: Tuberculosis (TB) is a major global public health problem. New treatment methods on TB are urgently demanded. In this study, Mycobacterium tuberculosis (MTB) rv2190c DNA vaccine was prepared and its immunogenicity and therapeutic effects were evaluated.

Results: Non-infected mice immunized with rv2190c DNA or ag85a DNA showed higher levels of interferongamma (IFN- $\gamma)$ in stimulated spleen lymphocyte culture supernatants, and had more Th1 cells and an elevatory ratio of Th1/Th2 immune cells in whole blood, indicating that Th1-type immune response was predominant. Compared with the saline group, ag85a DNA group and rv2190c DNA group in the infected mice decreased the lung colony-forming units (CFUs) by 0.533 and $0.283 \log _{10}$, and spleen CFUs by 0.425 and $0.321 \log _{10}$ respectively, and pathological lesion.
\end{abstract}

Conclusions: The rv2190c DNA had some immunotherapeutic effect on TB.

Keywords: DNA vaccine, rv2190c DNA, Immunotherapy, Mycobacterium tuberculosis

\section{Background}

Tuberculosis (TB) is a severe respiratory infectious disease in which protective immunity and pathological hypersensitivity to an intracellular bacterium coexist [1, 2]. In 2013, there were 9 million incident cases and 1.5 million deaths [3]. It is difficult to cure with anti-TB chemotherapy because a long treatment duration (a minimum of six-month chemotherapy) with multiple drugs was used on active $\mathrm{TB}$, many $\mathrm{TB}$ patients cannot complete a full period of treatment, which lead to failure of treatment and acquired drug resistance with eventual creation of multi-drugresistant TB (MDR-TB) [4]. Accordingly, effective vaccines are needed in the face of failing drug treatments, and this may include therapeutic vaccines.

\footnotetext{
* Correspondence: wu-xueqiong@263.net

${ }^{\dagger}$ Equal contributors

${ }^{1}$ Army Tuberculosis Prevention and Control Key Laboratory, Beijing Key Laboratory of New Techniques of Tuberculosis Diagnosis and Treatment, Institute of Tuberculosis Research, the 309th Hospital of Chinese PLA, Beijing 100091, People's Republic of China

Full list of author information is available at the end of the article
}

Protective immunity against TB is largely attributed to a cellular immune response in which the production of the Th1-type cytokines (for example interferon-gamma, IFN$\gamma)$ predominates over the production of the Th2-type cytokines (for example interleukin-4, IL-4) [5-7]. DNA vaccination has been found to establish and boost antigen-specific cellular immunity in the direction of responses. Furthermore, immunotherapy with plasmid DNA has been found to be an effective adjunctive treatment in combination with antibacterial chemotherapy in mice. It both shortened the period of treatment and improved the therapy outcome of latent TB infection $[8,9]$. Furthermore, we and others have shown that ag85a DNA vaccine can also have immunotherapeutic effects against MDR-TB in mice [10-12]. Here we report that the rv2190c gene of Mycobacterium tuberculosis (MTB) is similarly effective as a therapeutic DNA vaccine. The open reading frame (ORF) contains 1177 nucleotides and encodes a hypothetical protein with an NlpC/P60 domain [13]. Målen et al. [14] identified the protein product of $r \mathrm{v} 2190 \mathrm{c}$ in MTB 
culture filtrate, showing that it could be expressed and secreted in vitro. Parthasarathy et al. [15] showed that the gene and its protein product were essential for normal growth and virulence of MTB in vivo and McMurry et al. [16] found that Rv2190c peptide could stimulate peripheral blood mononuclear cells to secrete IFN- $\gamma$ in persons with latent $\mathrm{TB}$ infection. Beyond that, MTB Rv2190c antigen has not been extensively studied and thus therefore presents new possibilities for developing drug targets, diagnostic reagents and vaccines. Accordingly we evaluated its immunogenicity and immunotherapeutic effects as a DNA vaccine in mice.

\section{Methods}

Mice

One hundred 6-8 week age of female BALB/c mice without specific pathogen were purchased from the Academy of Military Medicine and Science, Beijing, China, maintained under infection barrier conditions in a negative pressure animal room in the $309^{\text {th }}$ Hospital of Chinese PLA, Beijing, China, and fed a sterile commercial mouse diet (Beijing KeAoXieLi Company Limited, China). The study procedures were approved by the 309th Hospital of the Chinese PLA Research Animal Ethics Committees.

\section{MTB strain}

MTB $\mathrm{H}_{37} \mathrm{Rv}$ was provided by National Institutes for Food and Drug Control, Beijing, China.

\section{Preparation of recombinant Rv2190c protein}

The procedures preparation of Rv2190c protein were briefly as follows: a 1168 bp gene fragment of $r v 2190 c$ was amplified by polymerase chain reaction (PCR). The forward primer with a Nhe I enzyme site: $5^{\prime}$ - CTAGCT AGCATGAGGCTCGACCAGAGGTGGTT -3'; the reverse primer with an EcoR I enzyme site: 5' - CCGGAA TTCTCAGTAACGGCGGGCGTCG - 3 '. The resulting PCR product was ligated with plasmid vector pET30a (Promega), and then transformed into E. coli DH5 $\alpha$ [17]. The recombinant plasmid $r v 2190 c / p E T 30 a$ was analyzed by Huada gene Ltd. Beijing, China and had 100\% identity with the designed sequence by BLAST analysis. The recombinant plasmid was transformed into $E$. coli strain BL21 (DE3). The expression and purification of recombinant Rv2190c protein was performed according to the method described previously [18].

\section{Construction of rv2190c DNA vaccine}

The construction map and method of rv2190c DNA vaccine were shown in Fig.1 and described previously [19]. The sequence encoding Rv2190c was amplified from MTB $\mathrm{H}_{37} \mathrm{Rv}$ by PCR. The forward primer with a Nde I enzyme site: 5' - CTAGGCTAGCCACCATGGGGCTCG ACCAGAGGTGGTT-3'; the reverse primer with an EcoR I enzyme site: 5' -CCGGAATTCTCA GTAACGGC GGGCGTCG -3' (synthesized by Shanghai Sangon Ltd. Beijing, China). The PCR product fragment was 1177 bp. Recombinant plasmid rv2190c DNA was sequenced by Huada gene Ltd. Beijing, China and was found to conform to the sequence designed using BLAST analysis. EndoFree plasmid purification kit (Qiagen, Hilden, Germany) was used to purify rv2190c DNA vaccine.

\section{Immunogenicity of rv2190c DNA vaccine}

Fifty female BALB/c mice were divided into 5 groups as follow: (1) saline as a negative control $(100 \mu \mathrm{l})$; (2) vector pVAX1 as a negative control (100 $\mu \mathrm{g}$ in $100 \mu \mathrm{l}$ saline); (3) M. vaccae $(22.5 \mu \mathrm{g}$ in $100 \mu \mathrm{l}$ saline, Longcome Biological Pharmacy, Anhui, China) as a positive control; (4) $a g 85 a$ DNA (100 $\mu$ g in $100 \mu \mathrm{l}$ saline), as a positive control; (5) rv2190c DNA (100 $\mu \mathrm{g}$ in $100 \mu \mathrm{l}$ saline), immunized intramuscularly three times at two-week intervals.

\section{Cytokine production in vitro}

The mice were sacrificed at three weeks after the third immunization. The mouse splenocytes were isolated and cultured $\left(5 \times 10^{5}\right.$ cells/well) with Ag85A or Rv2190c protein $(20 \mu \mathrm{g} / \mathrm{ml})$ or phytohaemagglutinin (PHA; $20 \mu \mathrm{g} / \mathrm{ml}$ ) for $72 \mathrm{~h}$. The levels of IFN- $\gamma$ and IL-4 in the splenocytes culture supernatants were detected using enzyme-linked immunosorbent assay (ELISA) kit (BD PharMingen, San Diego, California, USA) according to the manufacturer's procedures.

\section{Determination of $\mathrm{CD}^{+} \mathrm{T}$ cell subsets expressing intracellular IFN- $\gamma$ or IL-4}

The operation procedure was described previously [20]. Briefly, Th1 and Th2 cells responding to Ag85A or Rv2190c proteins were calculated. Cells expressing IFN$\gamma$ and IL-4 were presented as a percentage of the total population of $\mathrm{CD}^{+}$cells. The data were collected using a fluorescence-activated-cell-sorting (FACS) Calibur flow cytometer (BD Pharmingen) and analyzed using CellQuest software.

\section{Treatment of TB-infected mice}

Fifty mice were challenged with $6.4 \times 10^{5}$ colonyforming units (CFUs) of MTB $\mathrm{H}_{37} \mathrm{Rv}$ through trail vein injection, randomly divided into five groups mentioned above, and then treated at the third day after infection.

\section{Bacterium counts}

The mice were sacrificed by cervical dislocation under anesthetic with $5 \mathrm{ml}$ dethyl ether (Beijing Chemical 


\begin{abstract}
GAATTCTCAGTAACGGCGGGCGTCGTGGATCGGCCCCGACGAGTCCATCGGCACCAC CCGCACCGGAACACCGTAGGTGGAGGAATGAACCATAAGACCATCACCGATGTAGAT GCCT GCGTGTGACGCGTCGGAATAGAAGGTCAACGCGTCGCCGGGCTGCAGATCCGA CAACGCGACCGGCT GACCACCGT GAGCCAGCGCCTGGCT GGAGTGCGGTAACGCGAT ACCAGCCTGCTGGAACGCCCACATCACCAAGCCTGAGCAGTCGAACCCGCCGGGCGC GGCACCACCCCACGCGTAGGGCGCGCCGACCT GCGTCAACGCCGCTTGGACAACGGC CGTACGGTCGCCGCCAGCGCCGTCGGGCT GCACGAAAGGCAATCCGGGCATCCCACC AGGCGGCGGCGCCACGCCAGGCGCCGGGCCGTCGCCAGGCGGCGCACCGGGCGGCA ACGCCGCAGGTGGGGCCCCGGGGGCGATCGCAGCAACCGCCGGGACCGGTCCTGGAT CAGCGAGGGCCGTGCGCTCCTCCGGCGTCAACGCGACGTATTGCGACTTGACGACGG CAATCTGCACCTGCAGCTGGCTCTGTTTGTGCT GCAGATTCGCTCGTACCGCGGCAGC TTGCTCGGCCGCGGACCTGGCATCGGCCGCCGATTT GGCTGCAGCCTGCTCGGCCTTG ACGGCCTGTTCTCCAGCGGCCTTGAAACGGGCCATCT GCGTGGACATTTGAT GCGCCA TCACCCGCTGT ACCGATAGCCGATCGATCAACAGTTGCGGGGACTCCGCCGTCAGGA TCGCATCCAT GCCGTGGGTACGACCACCCATGTAGGTAGCGGCCGCGACCTTGTTCAC CGCCGTCTGAAAAGTCGCCAAGCGTGCTCTCGCAGCATCCAAGGCCGTTCTGTT GTCC GCAAGCTTCTGGTCGGCGGCCCGCTGGGCAGCGAGCTTTTCGTTGAGATCCAGCTGCG CACT GTGCAGCGCCTCGGTGGT CTGCTCGGCCTGCCGGGATAACT CGTTGAGCTTGGC CAGCGCGTCGTCGGCCGGATCAGCCAGCACATTCGCGGCCAGGACGCCGGAGGAGAC GGTGAAGCTCGCAAAGAAACCTAT GGCGGACCGCATGATTACACGCGCGATCAACCA
\end{abstract} CCTCTGGTCGAGCCGCTAGC
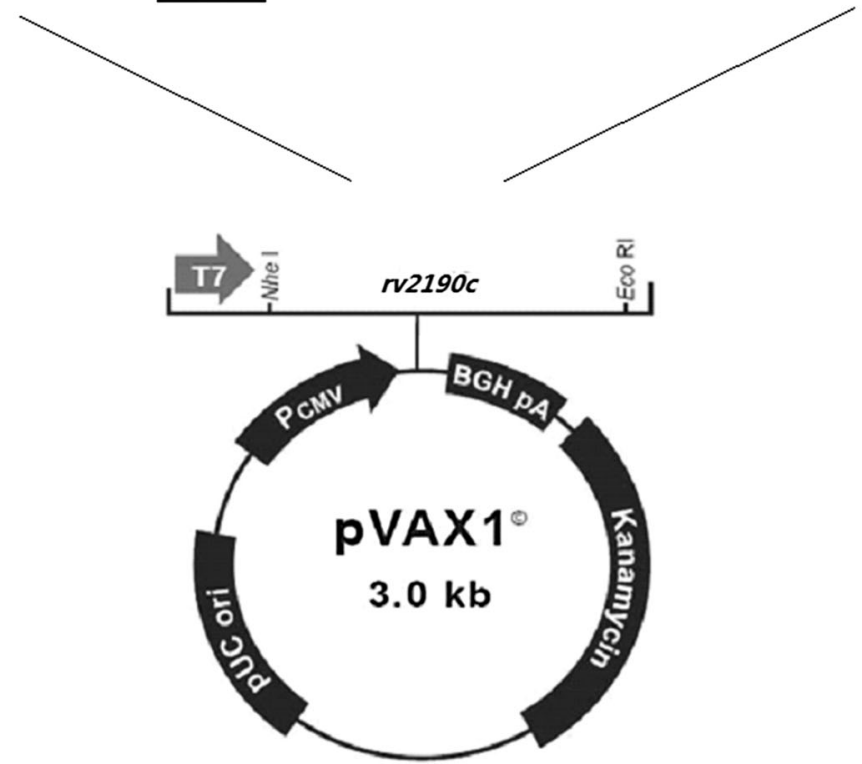

Fig. 1 The construction map and gene sequence of rv2190c DNA. Black letters are the rv2190c gene sequence. The bold and underline letters are the site of restrictive enzymes Nhe I and EcoR I. The double underline letters are the optimized gene sequence

Reagents Company, Beijing, China) at two weeks after the third immunization. The tissue suspensions of mouse lungs and spleens were serially diluted 10 -fold, and $100 \mu \mathrm{L}$ suspension dilution were inoculated in duplicate on Lowenstein-Jensen medium plates and cultured at $37^{\circ} \mathrm{C}$ for 4 weeks. MTB colonies on medium were counted and the results were showed as CFUs per organ.

\section{Lung histopathological examination}

The mouse lungs tissues paraffin-embedded were sliced into $3-\mu \mathrm{m}$ thick tissue sections, which were dyed with hematoxylin and eosin, and then examined by a certified and veteran pathologist.

\section{Statistical analyses}

Data are shown as means and standard deviations. Statistical analyses were performed using one-way ANOVA followed by Dunnett's multiple comparison test, and a $P$-value of $<0.05$ was considered as significant difference.

\section{Results}

Preparations of rv2190c DNA and recombinant Rv2190c protein

The nucleotide sequence in pVAX1-rv2190c plasmid had $100 \%$ identity with MTB $r v 2190 c$ sequence as designed and the fragment size from restriction-enzyme- 
digested recombinant plasmid pVAX1-rv2190c was $1.177 \mathrm{~kb}$ on $0.8 \%$ agarose gel electrophoresis, and that confirmed the successful construction (Additional file 1: Figure S1).

The recombinant Rv2190c protein was soluble in expression and amounted to 30\% of total bacterial protein. The molecular mass of the purified Rv2190c protein was around $42 \mathrm{kDa}$ by SDS-PAGE, its purity was higher than 90\% (Additional file 2: Figure S2).

\section{Specific cytokine production levels in splenic lymphocyte culture supernatants}

The IFN- $\gamma$ level in splenic lymphocyte culture supernatants in the rv2190c DNA group was obviously higher than those in the saline, plasmid vector and $M$. vaccae groups $(P<0.05)$, but had no significant difference from that in the ag85a DNA group. The production of IL-4 was not significantly different between groups (Fig.2, Additional file 3).

\section{$\mathrm{CD4}^{+} \mathrm{T}$ cell subsets expressing intracellular IFN- $\gamma$ or IL-4} The proportion of CD4+ T cells expressing IFN- $\gamma$ (Th1) in response to Ag85A or Rv2190c proteins was significantly higher in the whole blood from the rv2190c DNA group than those from the saline group and vector group by flow cytometry $(P<0.001)$, but there was no significant difference from the $M$. vaccae group or ag85a DNA group. The proportion of cells expressing IL-4 (Th2) was significantly higher in the blood from the $r v 2190 c$ DNA group than those from the control groups $(P<0.05)$, but there was no significant difference from the $M$. vaccae group or ag85a DNA group. Th1/Th2 ratio in the blood from the rv2190c DNA group was significantly decreased than that in the ag85a DNA group, and increased than those in the saline, vector and
$M$. vaccae groups, but the differences were not significant (Fig. 3, Additional file 3).

\section{Mouse survival}

There was one mouse death in vector group, one in $M$. vaccae group and one in $r v 2190 c$ DNA group at 29 days after infection ( $90 \%$ survival). The other mice were all alive.

\section{Bacterial counts in the lungs and spleens}

The live bacteria in mouse lungs and spleens were determined at two weeks after third of immunotherapy. The lung CFUs from saline, vector, $M$. vaccae, ag85a DNA and $r v 2190 c$ DNA groups were $7.334 \pm 0.180,7.233 \pm$ $0.102, \quad 7.081 \pm 0.369, \quad 6.801 \pm 0.407$ and $7.051 \pm 0.154$ $\log _{10}$, respectively (Fig.4a, Additional file 3), and the spleen CFUs were $6.919 \pm 0.117,6.808 \pm 0.067,6.652 \pm$ $0.345,6.494 \pm 0.211$ and $6.598 \pm 0.143 \log _{10}$, respectively (Fig.4b, Additional file 3). Compared with saline group, $r v 2190 c$ DNA and ag85a DNA decreased the lung CFUs by $0.283(P>0.05)$ and $0.533 \log _{10}(P<0.05)$ and the spleen CFUs by 0.321 and $0.425 \log _{10}(P<0.05)$, respectively.

\section{Histopathological changes}

The lung sections from the saline group and plasmid vector group showed extensive lung lesions, in which hyperemia and congestion in alveoli with many lymphocytes and destructive structure caused by severe inflammation. The lung sections from treatment groups showed more foamy macrophages but less lymphocyte infiltration, the alveoli were relatively clear and had normal structure. Representative histopathological changes of five groups were shown in Fig.5.
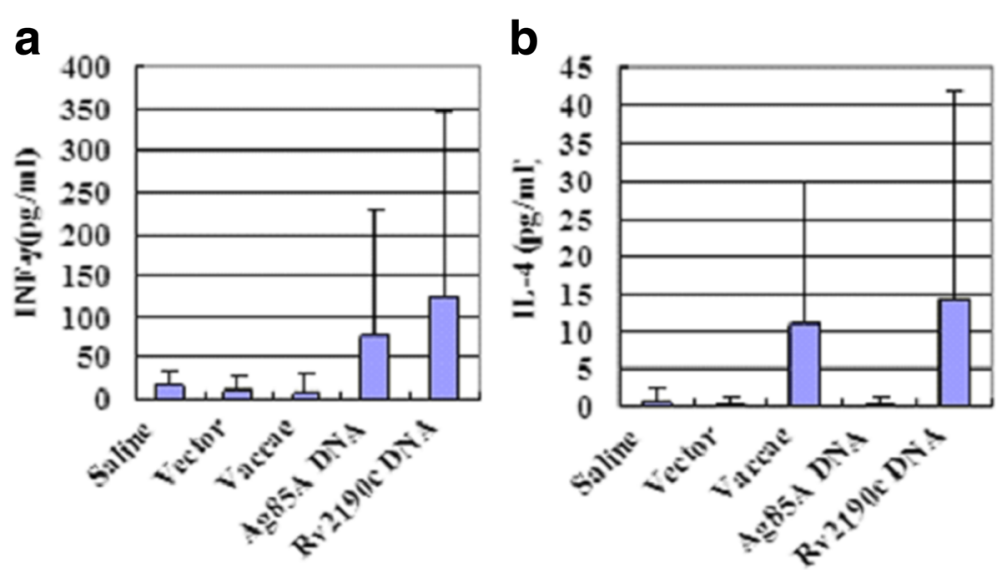

Fig. 2 IFN- $\gamma$ (a) and IL-4 (b) levels in the culture supernatants of splenocytes were detected by ELISA. The production of IFN- $\gamma$ from rV2190c DNA group was significantly higher than from the saline group, vector group and $M$. vaccae group $(P<0.05)$, but the production of IL-4 was not significantly different between the groups 

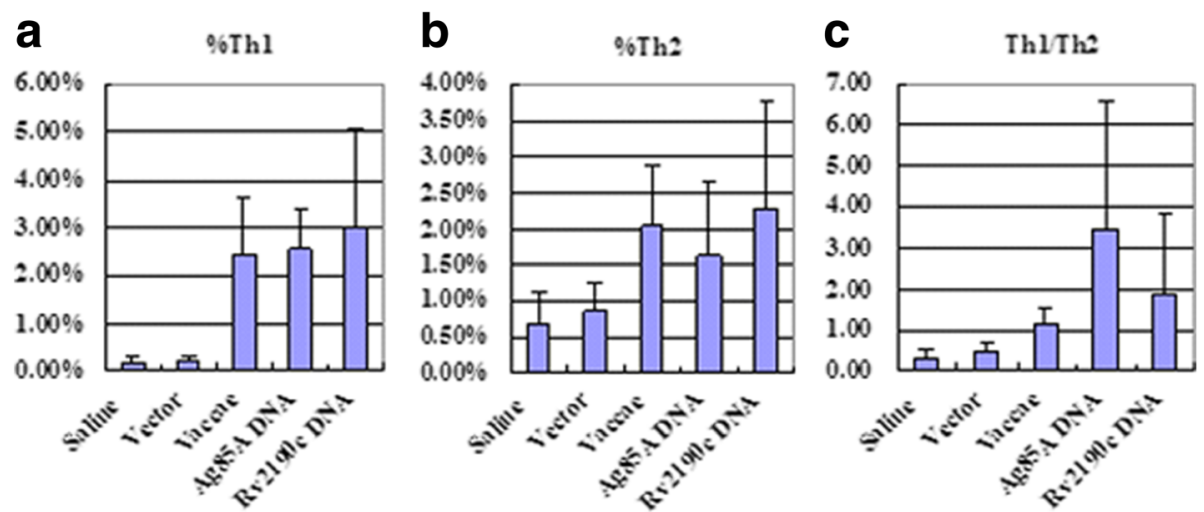

Fig. 3 Frequencies of CD4+ T cell subsets in whole blood assessed by flow cytometry. The data are expressed as the mean $\% \pm s t a n d a r d$ deviation. a Th1 cells expressing IFN- $\gamma$ (IFN- $\gamma$-FITC); b Th2 cells expressing IL-4 (IL-4-PE); c The ratio of Th1/Th2 cell frequencies

\section{Discussion}

In this study, a DNA sequence encoding MTB Rv2190c protein was inserted into plasmid vectors pET30a and pVAX1, thereby recombinant Rv2190c protein and $r v 2190 c$ DNA vaccine were prepared and subsequently used to compare the therapeutic effects of $r v 2190 c$ DNA with those of ag85a DNA as vaccines against TB in mice.

The cellular immune responses to rv2190c DNA were measured as increase in frequency of circulating $\mathrm{T}$ cells that produced either IFN- $\gamma$ or IL- 4 in response to $\operatorname{cog}$ nate vaccine antigen since these cytokines are markers of protective Th1 responses and non-protective Th2 responses respectively. A balance between Th1 and Th2 in which Th1 predominates is essential for the control of $\mathrm{TB}$ and mycobacterial infection in mice and human $[2,4]$. In this study, the significantly increased IFN- $\gamma$ level in the spleen lymphocyte culture supernatant, the abundance of Th1 cells and elevatory ratios of Th1 /Th2 cells in antigen-specifically stimulated whole blood from the rv2190c DNA group were all similar to the results obtained in the ag85a DNA group. Thus both vaccines induced a predominantly Th1 immune response [5, 12-14]. This finding was consistent with evidence from McMurry et al. [16] showing that Rv2190c peptide can induce IFN- $\gamma$ production in cells from persons with latent TB infection, and indicated a potential utility of Rv2190c antigen in therapeutic vaccination.

When used to treat infected mice, rv2190c DNA vaccine did indeed reduce the numbers of live bacteria found in the organs sampled after 6 weeks of treatment when compared to control mice. However, the reduction in lung bacterial load was small, not statistically significant, and less than that obtained after ag85a DNA treatment. In contrast, the reduction of load in the spleen was significant, but again less than that observed after ag85a DNA treatment. Apparently, although the rv2190c protein enhanced antibacterial immunity it was in this respect a less effective vaccine antigen than the
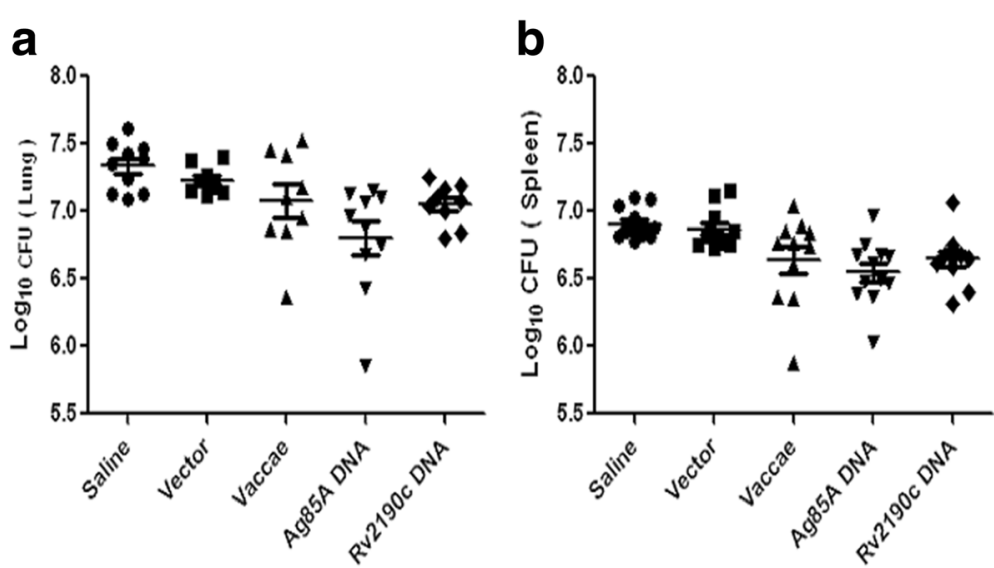

Fig. 4 The numbers of live bacteria in lungs (a) and spleens (b) at six weeks after infection. Compared with the saline group, rv2190c DNA and ag85a DNA reduced the pulmonary bacterial loads by $0.283(P>0.05)$ and 0.533 logs $(P<0.05)$ and the splenic bacterial loads by $0.321(P<0.05)$ and $0.425 \operatorname{logs}(P<0.05)$, respectively 

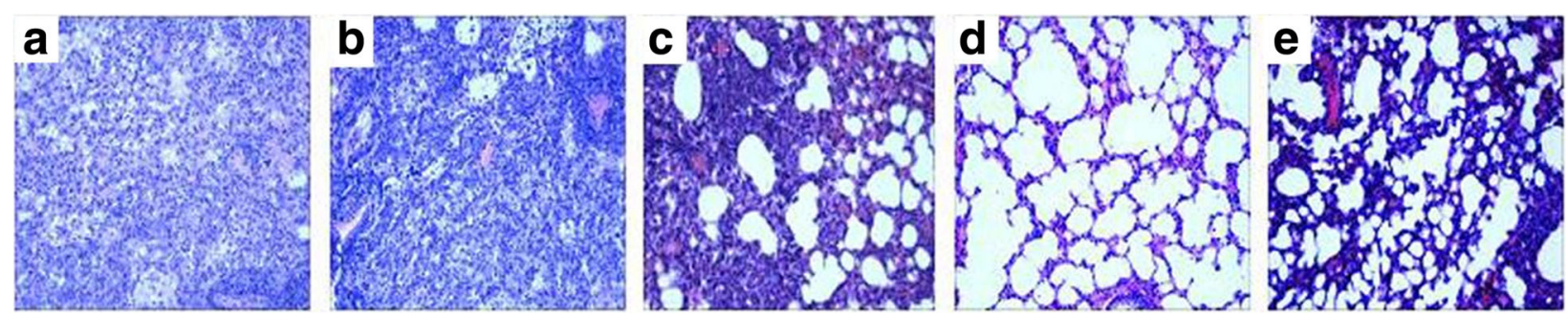

Fig. 5 Pulmonary histopathological changes. This figure shows representative photomicrographs $(H \& E, 100 x)$ of lung tissue obtained from mice each group at six weeks after infection with MTB H37Rv. a Saline group. b Vector group. c M. vaccae group. d ag85a DNA group. e rv2190c DNA group

Ag85A protein. Nevertheless, in a practical therapeutic vaccine for human use, more than one antigen is likely to be required and Rv2190c is clearly a candidate for inclusion. The greater effects in spleen than in lung suggest that the DNA vaccines were more active against bacteria in extra-pulmonary sites than in the lung, but whether this was due to differential effects on bacterial dissemination, growth inhibition or killing was not investigated. Although organ bacterial load is one of the important indicators to evaluate curative effects on animal TB experiments [21], evaluation of the impact upon pathology is also essential. Strikingly, the lesions in the lungs of rv2190c DNA and ag85a DNA vaccinated groups were similarly lessened, suggesting that $r v 2190 c$ DNA and ag85a DNA provided similarly efficient immunotherapy for TB disease in this model.

\section{Conclusions}

We successfully constructed a MTB rv2190c DNA vaccine that could induce Th1-type cellular immune reactions in mice and had some immunotherapeutic effects on tuberculosis in mice. It may have potential for use in an immunotherapeutic DNA vaccine against TB.

\section{Additional files}

Additional file 1: Figure S1. Fragment sizes of restriction-enzymedigested recombinant plasmid pVAX1-rv2190c. 1. PCR amplification product of the recombinant plasmid $p V A X 1-r v 2190 c$ colony; 2 . The recombinant plasmid pVAX1-rV2190c digested by restriction endonuclease Nhe I and EcoR 1; 3. Digest of the pVAX1 vector DNA with restriction endonuclease Nhe I and EcoR l; 4. The recombinant plasmid pVAX1-rV2190c DNA digested by restriction endonuclease EcoR I; 5. Digest of the PVAX1 Vector DNA with restriction endonuclease EcoR I; M: DM5000 DNA Marker. (TIF 45 kb)

Additional file 2: Figure S2. Expression and purification of recombinant Rv2190c protein as determined by SDS-PAGE electrophoresis. The gel was subjected to electrophoresis followed by Coomassie blue staining. Lane M, protein molecular weight marker. Lane 1, E. coli lysates engineered before isopropylthiogalactopyranoside (IPTG) induction. Lane 2, E. coli lysates engineered after induction with IPTG. Lane 3, the purified recombinant Rv2190c protein. (TIF $39 \mathrm{~kb}$ )

Additional file 3: The Excel data file [FOLT] Figshare, [DOl:10.6084/ m9.figshare.4668148 and https://figshare.com/s/bd46c22986c673579bb6] includes all datasets supporting the conclusions of this article: IFN- $\gamma$ in spleen lymphocyte culture supernatants, LL-4 in spleen lymphocyte culture supernatants, CD4+ T cell subsets expressing intracellular IFN- $\gamma$ or IL-4, CFU in the lungs and spleens.. (XLS $143 \mathrm{~kb}$ )

\section{Abbreviations}

CFUs: Colony-forming units; ELISA: Enzyme-linked immunosorbent assay; FACS: Fluorescence-activated-cell-sorting; IFN- $\gamma$ : Interferongamma; IL-4: Interleukin-4; MDR-TB: Multi-drug-resistant tuberculosis; MTB: Mycobacterium tuberculosis; ORF: Open reading frame; PCR: Polymerase chain reaction; TB: Tuberculosis

\section{Funding}

This project was supported by the grant from the Serious Infectious Diseases Special Foundation of China (2012ZX10003008002).

\section{Availability of data and materials}

The data that support the findings of this study are included in the main manuscript and supplemetary file.

\section{Authors' contributions}

$Y L$ participated in designing and performing the experiments, writing the manuscript. XZ participated in the construction of rv2190c DNA vaccine, tested immunogenicity of $r 2190$ c DNA vaccine and drafted the manuscript. XB carried out the preparation of recombinant Rv2190c protein. LX carried out the immunoassays. XW and LW participated in the animal experiments. $J Z$ and YY carried out the statistical analysis. JS performed the lung histopathological examination. XW designed the study, directed the experiments and revised the manuscript. All authors approved this manuscript.

\section{Competing interests}

The authors declare that they have no competing interests.

\section{Consent for publication}

The authors have stated that it is not applicable.

\section{Ethics approval}

The study procedures were approved by the 309th Hospital of the Chinese PLA Research Animal Ethics Committees.

\section{Author details}

${ }^{1}$ Army Tuberculosis Prevention and Control Key Laboratory, Beijing Key Laboratory of New Techniques of Tuberculosis Diagnosis and Treatment, Institute of Tuberculosis Research, the 309th Hospital of Chinese PLA, Beijing 100091, People's Republic of China. '2 Zhengzhou Kingmed Center for Clinical Laboratory, Zhengzhou 450016, People's Republic of China.

Received: 4 June 2016 Accepted: 16 February 2017

Published online: 27 February 2017

References

1. Kaufmann SHE. Immunity to intracellular bacteria. Annu Rev Immunol. 1993; 11:129-63. 
2. Paul WE, Seder RA. Lymphocyte responses and cytokines. Cell. 1994;76: 241-51.

3. World Health Organization. Global tuberculosis report 2014. Geneva: WHO; 2014. Report No:: WHO/HTM/TB/2014.08.

4. Manabe YC, Bishai WR. Latent Mycobacterium tuberculosis-persistence, patience, and winning by waiting. Nat Med. 2000;6:1327-9

5. Cooper AM, Dalton DK, Stewart TA, Griffin JP, Russell DG, Orme IM. Disseminated tuberculosis in interferon gamma gene disrupted mice. J Exp Med. 1993;178:2243-7.

6. Flynn JL, Chan J, Triebold KJ, Dalton DK, Stewart TA, Bloom BR. An essential role for interferon gamma in resistance to Mycobacterium tuberculosis infection. J Exp Med. 1993;178:2249-54.

7. Flynn JL. Immunology of tuberculosis and implications in vaccine development. Tuberculosis. 2004;84:93-101.

8. Ha SJ, Jeon BY, Kim SC, Kim DJ, Song MK, Sung YC, Cho SN. Therapeutic effect of DNA vaccines combined with chemotherapy in a latent infection model after aerosol infection of mice with Mycobacterium tuberculosis. Gene Ther. 2003;10:1592-9.

9. Yu D, Hu X, Cai H. Efficient tuberculosis treatment in mice using chemotherapy and immunotherapy with combined DNA vaccines encoding Ag85B, MPT64 and MPT83. Gene Ther. 2008;15:652-9.

10. Liang Y, Wu X, Zhang J, Li N, Yu Q, Yang Y, et al. The treatment of mice infected with multi-drug-resistant Mycobacterium tuberculosis using DNA vaccines or in combination with rifampin. Vaccine. 2008;26:4536-40.

11. Liang Y, Wu X, Zhang J, Yang Y, Wang L, Bai X, et al. Treatment of multidrug-resistant tuberculosis in mice with DNA vaccines alone or in combination with chemotherapeutic drugs. Scand I Immunol. 2011;74:42-6.

12. Ha SJ, Jeon BY, Youn JI, Kim SC, Cho SN, Sung YC. Protective effect of DNA vaccine during chemotherapy on reactivation and reinfection of Mycobacterium tuberculosis. Gene Ther. 2005;12:634-8.

13. Fleischmann RD, Alland D, Eisen JA, Carpenter L, White O, Peterson J, et al. Whole-genome comparison of Mycobacterium tuberculosis clinical and laboratory strains. J Bacteriol. 2002:184:5479-90.

14. Målen H, Berven FS, Fladmark KE, Wiker HG. Comprehensive analysis of exported proteins from Mycobacterium tuberculosis H37Rv. Proteomics. 2007; 7:1702-18.

15. Parthasarathy G, Lun S, Guo H, Ammerman NC, Geiman DE, Bishai WR Rv2190c, an NlpC/P60 family protein, is required for full virulence of Mycobacterium tuberculosis. PLoS One. 2012;7:e43429.

16. McMurry J, Sbaia H, Gennaro ML, Carter EJ, Martin W, De Groot AS. Analyzing Mycobacterium tuberculosis proteomes for candidate vaccine epitopes. Tuberculosis. 2005;85:95-105.

17. Sambrook J, Fritsch EF, Maniatis T. Molecular cloning: a laboratory manual. 2nd ed. New York: Cold Spring Harbor Laboratory Press; 1989.

18. Wu X, Yang $Y$, Han $Y$, Zhang J, Liang $Y$, Li H, et al. Effect of recombinant Rv1009 protein on promoting the growth of Mycobacterium tuberculosis. J Appl Microbiol. 2008;105:1121-7.

19. Liang $Y$, Zhang $X$, Xiao L, Bai X, Wang X, Yang $Y$, et al. Immunogenicity and therapeutic effects of pVAX1- rv1419 DNA from Mycobacterium tuberculosis. Curr Gene Ther. 2016. doi : 10.2174/1566523216666161102170123.

20. Liang Y, Wu X, Zhang J, Xiao L, Yang Y, Bai X, et al. Immunogenicity and therapeutic effects of $A g 85 A / B$ chimeric DNA vaccine in mice infected with Mycobacterium tuberculosis. FEMS Immunol Med Microbiol. 2012;66:419-26.

21. Lowrie DB, Tascon RE, Bonato VLD, Lima VMF, Faccioli LH, Stavropoulos E, Colston MJ, Hewinson RG, Moelling K, Silva CL. Therapy of tuberculosis in mice by DNA vaccination. Nature. 1999:400:269-71.

\section{Submit your next manuscript to BioMed Central and we will help you at every step:}

- We accept pre-submission inquiries

- Our selector tool helps you to find the most relevant journal

- We provide round the clock customer support

- Convenient online submission

- Thorough peer review

- Inclusion in PubMed and all major indexing services

- Maximum visibility for your research

Submit your manuscript at www.biomedcentral.com/submit
Biomed Central 